\section{Tuberkulose: Minimale Hemmkonzentration vorher testen?}

Colangeli R et al. Bacterial Factors That Predict Relapse after Tuberculosis Therapy. N Engl J Med 2018; 379: $823-833$

Etwa 5\% der Patienten mit Tuberkulose, die auf eine medikamentöse Therapie ansprechen, erleiden innerhalb von 6 Monaten nach der Erstlinientherapie ein Rezidiv, bei Kurzzeittherapie sind es sogar $20 \%$ nach 4 Monaten. Robert Clangeli, Newark (USA), et al. untersuchten, ob eine minimale Hemmkonzentration (MIC) unter der Standardresistenzschwelle der Mycobacterium-tuberculosis-Isolate vor Therapie mit dem Rezidivrisiko nach Therapie korreliert.

Die Untersuchung führten die Teams der beiden Studien DMID 01-009 und Tuberculosis Trials Consortium Study 22 durch. Die Daten von 1004 Patienten aus der Tuberculosis Trials Consortium Study 22 dienten als Entwicklungskohorte. An ihr wurden Isolate von später geheilten Patienten und solchen mit Rezidiv untersucht, um die prätherapeutischen MICWerte von Isoniazid und Rifampin unterhalb der Standardresistenzschwelle $(0,1$ $\mu \mathrm{g} / \mathrm{ml}$ bei Isoniazid und $1,0 \mu \mathrm{g} / \mathrm{ml}$ bei Rifampin) zu bestimmen. Unter Zuhilfenahme von klinischen, radiologischen und Laborparametern wurde dann ein prädiktives Modell für die Rezidivwahrscheinlichkeit entwickelt, das anhand der Daten aus der DMID 01-009 Studie validiert wurde.

\section{Ergebnisse}

In der Entwicklungskohorte lag die mittlere MIC unter der Isoniazid-Resistenzschwelle in der Gruppe von Patienten mit Rezidiv bei 0,0334 $( \pm 0,0085) \mu \mathrm{g} / \mathrm{ml}$, in der Gruppe mit geheilten Patienten bei $0,0286( \pm 0,0092) \mu \mathrm{g} / \mathrm{ml}$. Damit lag die MIC in der Rezidivgruppe um den Faktor 1,17 höher $(p=0,02)$. Die entsprechenden MIC-Werte für Rifampin lagen bei 0,0695 $( \pm 0,0276)$ und 0,0453 $( \pm 0,0223) \mu \mathrm{g} / \mathrm{ml}$, was einer um den Faktor 1,3 höheren MIC bei späterem Rezidiv entspricht $(p<0,001)$. Eine multivariable Analyse, die auch andere Unterschiede in den Gruppen berücksichtigte, bestätigte die Assoziation von höheren MICWerten und späterem Rezidiv. Eine Analyse der Grenzwertoptimierungskurve der Rezidive basierend auf diesen MICWerten ergab eine Fläche unter der Kurve (Area under the curve; AUC) von 0,779. Wurden weitere Einflussfaktoren einbezogen, ergab sich in der Entwicklungskohorte eine AUC von 0,875. In der Validierungskohorte lag die AUC auf Basis der MIC vor Therapie alleine bei 0,929 , im multivariablen Modell bei 0,929 . Die Sensitivität des Modells erreichte in der Validierung eine $75 \%$ ige Sensitivität und eine 76,5\%ige Spezifität in der Entwicklungskohorte und eine Sensitivität von 70,0\% und eine Spezifität von $100 \%$ in der Validierungskohorte.

\section{FAZIT}

Der Unterschied zwischen den MICWerten von Isoniazid und Rifampin von Mycobacterium-tuberculosis-Isolaten vor Therapiebeginn und den Standardresistenzschwellen der beiden Therapeutika hat einen Einfluss auf das Behandlungsergebnis, schlussfolgern die Autoren. Sie betonen aber auch, dass diese Befunde noch in größeren prospektiven Kohorten bestätigt werden müssen, bei denen bereits vor Behandlungsbeginn die MIC der Isolate bestimmt werden und nicht retrospektiv.

Friederike Klein, München 